\title{
Comparison between nonlinear measurements in patterned and unpatterned thin films
}

\author{
C Collado' ${ }^{1}$ J Mateu ${ }^{2}$ and J M O'Callaghan ${ }^{1}$ \\ ${ }^{1}$ Department of Signal Theory and Communications, Universitat Politècnica de Catalunya, \\ Campus Nord UPC, D3-Jordi Girona, 1-3, 08034 Barcelona, Spain \\ ${ }^{2}$ Centre Tecnològic de Telecomunicacions de Catalunya, Edifici Nexus Gran Capità, 2-4, \\ 2nd floor, room 202-203, 08034 Barcelona, Spain \\ E-mail: jordi.mateu-mateu@cttc.upc.es and joano@tsc.upc.es
}

Received 5 December 2003

Published 18 May 2004

Online at stacks.iop.org/SUST/17/876

DOI: 10.1088/0953-2048/17/7/009

\begin{abstract}
This work compares two alternative methods of characterizing the nonlinearities in a $10 \times 10 \mathrm{~mm}^{2}$ superconducting thin film. Both methods are based on measuring the intermodulation distortion in high temperature superconducting (HTS) films. The first method measures the unpatterned film by using a rutile loaded cavity operating at the $\mathrm{TE}_{011}$ mode. The second method is based on intermodulation measurements made in a resonant coplanar line which is patterned on the same film that is used in the rutile cavity. In both experiments we use closed-form expressions and numerical techniques to extract local parameters related to the nonlinearities of the superconductor. The intermodulation data in both type of measurements can be fitted with identical nonlinear parameters of the HTS.
\end{abstract}

\section{Introduction}

Early in the application of HTS materials it was noticed that they exhibit a surface impedance that is dependent on the applied field [1]. The origin of these nonlinearities is not yet fully understood. To overcome this, many experiments have been carried out over the last decade, some of which still remain unexplained. Most of the experimental work has been done measuring intermodulation products in planar patterned devices [2], but experiments have also been done on unpatterned thin films [3].

Intermodulation measurements on patterned devices are interesting since most practical devices (like filters) are based on planar patterned structures. In this case the nonlinearities might come from many causes-HTS properties, possible damage due to patterning, shape of resonator, etc, which need to be characterized. This raises the question of how representative of HTS properties are the experiments performed on patterned samples. To address this it is necessary to compare intermodulation measurements of films before and after patterning. This should be done on a large number of representative HTS samples using a procedure that allows a quantitative comparison between the patterned and unpatterned measurements.

In this work we propose such a procedure, which is based on obtaining parameters describing the HTS nonlinearities from intermodulation measurements made on unpatterned and patterned thin films. As an example of the procedure, we have characterized the nonlinearities in a $10 \times 10 \mathrm{~mm}^{2}$ YBCO thin film on $\mathrm{MgO}$, by measuring the intermodulation products with a rutile-loaded cavity operating at $\mathrm{TE}_{011}$ mode [4] and a coplanar half-wave resonator.

\section{Models for the nonlinear characterization of HTS materials}

The nonlinearities can be quantified by a relative change of the penetration depth, $\lambda_{L}$, on the volume current density, $j$, as:

$$
\lambda_{L}(T, j)=\lambda_{L}(T, 0)\left[1+\frac{1}{2} f(T, j)\right]
$$

where $f(T, j)$ sets how the penetration depth deviates from its small signal value (i.e. $f(T, 0)=0$ ). Several 
references $[5,6]$ use $f(T, j)=\left(j / j_{\mathrm{IMD}}\right)^{2}$ to be consistent with their intermodulation measurements, but others claim other types of dependence like, $f(T, j)=\left|j / j_{\mathrm{IMD}}\right|[3,7]$ or $f(T, j)=\left|j / j_{\mathrm{IMD}}\right|^{0.2}$ [3]. In order to deal with the various types of dependence observed, we have developed a formulation that assumes a generic variation of the penetration depth on the volume current density [8]:

$$
f(T, j)=\left|\frac{j}{j_{\mathrm{IMD}, \alpha}(T)}\right|^{\alpha}
$$

where $\alpha$ and $j_{\mathrm{IMD}, \alpha}$ are empirically determined parameters that set the nonlinearities and can be obtained by fitting the data from intermodulation or other nonlinear measurements.

Equation (1) allows us to relate the volume current density with the electric field in the superconductor. However, there are situations where it would be useful to describe the electric field at the surface as a function of the surface current density $\left(j_{\mathrm{s}}\right)$. This happens, for example, in cavities where superconductors are used as endplates.

Hence, for modelling the nonlinearities on the superconductor we will assume that the electric field at the surface of the superconductor is the sum of a linear contribution plus an additional electric field $e_{\mathrm{NL}}$ caused by the nonlinearities. In this work (and as we did in [8]) we will use:

$$
\vec{e}_{\mathrm{NL}}=\Delta R_{\alpha}\left|j_{\mathrm{s}}\right|^{\alpha} \vec{j}_{\mathrm{s}}+\frac{\mathrm{d}}{\mathrm{d} t}\left(\Delta L_{\alpha}\left|j_{\mathrm{s}}\right|^{\alpha} \vec{j}_{\mathrm{s}}\right)
$$

where $\alpha, \Delta R_{\alpha}$ and $\Delta L_{\alpha}$ are parameters that characterize the strength of the nonlinearity in the HTS.

\section{Formulation for IMD distortion of a resonator}

In a resonator, the current at the surface of the HTS film can be written as $\vec{j}_{\mathrm{s}}(\vec{r}, t)=A(t) f(\vec{r}) \hat{u}(\vec{r})$, where $\hat{u}(\vec{r})$ is a unit vector and $f(\vec{r}) \hat{u}(\vec{r})$ describes the spatial dependence of the current density. $A(t)$ describes the time dependence which, for the case of an intermodulation experiment, will consist of the sum of two sinusoidal signals with amplitudes $j_{\mathrm{s}, 1}$ and $j_{\mathrm{s}, 2}$, thus $A(t)=j_{\mathrm{s}, 1} \cos \omega_{1} t+j_{\mathrm{s}, 2} \cos \omega_{2} t$.

The expansion to find the intermodulation products coupled out of the resonator is fully detailed in [8] and [9]. If we assume that the device is fed with input signals whose frequencies are very similar $\omega_{1} \approx \omega_{2} \approx 2 \omega_{1}-\omega_{2}$ (which we will denote as $\omega_{0}$ ), the power coupled out of the resonator at the third order intermodulation product $2 \omega_{1}-\omega_{2}$ will be:

$$
\begin{aligned}
P_{L} & =\left(4 P_{0, \omega_{1}}\right)^{\alpha}\left(4 P_{0, \omega_{2}}\right)\left(\frac{Q_{0}}{\omega_{0} W_{0}}\right)^{\alpha+2}\left|\left(\Delta R_{\alpha}+j \omega_{0} \Delta L_{\alpha}\right)\right|^{2} \\
& \times K T_{\alpha, 2 \omega_{1}-\omega_{2}}^{2} \Gamma_{\alpha}^{2},
\end{aligned}
$$

where $P_{0, \omega_{1}}$ and $P_{0, \omega_{2}}$ are the available powers at fundamental frequencies and $Q_{0}$ is the unloaded quality factor. The term $T_{\alpha, 2 \omega_{1}-\omega_{2}}$ quantifies the intermodulation produced by the function $|A(t)|^{\alpha} A(t)$ at $2 \omega_{1}-\omega_{2}$ and depends on $\alpha$ and on the quotient $j_{1} / j_{2}$ and can be calculated from equation (8) of [8]. The term $\Gamma_{\alpha}$ is equal for all the frequency components at resonance and is only dependent on $f(\vec{r})$ and on $\alpha$ [8]. $K$ is a coupling factor which is equal to $\left(\kappa_{1} /\left(1+\kappa_{1}\right)^{2}\right)^{\alpha+2}$ for oneport resonators and is equal to $\left(\kappa_{2} \kappa_{1}^{(\alpha+1)}\right) /\left(1+\kappa_{1}+\kappa_{2}\right)^{2(\alpha+2)}$ for two-port resonators, $k_{i}$ being the coupling factor of the $i$-port.
Finally, $W_{0}$ is the normalized stored energy in the resonator at $2 \omega_{1}-\omega_{2}$ with respect to the magnetic field amplitude at the surface.

Equation (4) is important since it relates the measurable quantity $P_{L}$ (measured power of the intermodulation products) to the HTS nonlinear parameters: $\left|\Delta R_{\alpha}+j \omega_{0} \Delta L_{\alpha}\right|$ and $\alpha$. To apply (4), the normalized field distribution of the resonant mode $f(\vec{r})$ and the values of $Q_{0}$ and $k_{i}$ have to be known.

\subsection{Dielectric loaded cavity}

We apply the general analysis outlined above for a $\mathrm{TE}_{011}$ dielectric-loaded resonator cavity [3]. For nonlinear characterization by measuring intermodulation products, unlike in surface resistance measurements, one of the endplates in our cavity may be a normal metal since it is not required that the HTS losses dominate the overall cavity losses. This has the advantage of making the nonlinear properties of the cavity dependent on the nonlinearities of a single HTS film at the expense of reducing the circulating power and the power of the intermodulation products.

In these conditions, the surface current density of the HTS endplate in an intermodulation experiment can be written as $\vec{j}_{\mathrm{s}}(\rho, t)=\left(j_{\mathrm{s}, 1} \cos \omega_{1} t+j_{\mathrm{s}, 2} \cos \omega_{2} t\right) f(\rho) \hat{\phi}$, where $\hat{\phi}$ is the unit vector in the azimuthal direction and $f(\rho)$ describes the radial dependence of the $\mathrm{TE}_{011}$ mode (see [10] for a detailed expression). Reference [8] shows the steps to find the parameter $\Gamma_{\alpha}$ to be used in (4):

$$
\begin{aligned}
\Gamma_{\alpha} & =\pi\left[\left|\frac{\beta}{\xi_{1}}\right|^{\alpha+2} \int_{0}^{a}\left|J_{1}\left(\xi_{1} \rho\right)\right|^{\alpha+2} \rho \mathrm{d} \rho\right. \\
& \left.+\left|\frac{\beta}{\xi_{2}} \frac{J_{0}\left(\xi_{1} a\right)}{F_{0}\left(\xi_{2} b\right)}\right|^{\alpha+2} \int_{a}^{b}\left|F_{1}\left(\xi_{2} \rho\right)\right|^{\alpha+2} \rho \mathrm{d} \rho\right]
\end{aligned}
$$

where $a$ is the radius of the dielectric, $b$ is the radius of the metal cavity, $\beta$ is the $z$-direction propagation constant, and $\xi_{1}$ and $\xi_{2}$ are the $\rho$-direction wavenumbers (inside and outside the dielectric respectively). The functions $F_{0}\left(\xi_{2} \rho\right)$ and $F_{1}\left(\xi_{2} \rho\right)$ can be written as [10]: $F_{0}\left(\xi_{2} \rho\right)=$ $I_{0}\left(\xi_{2} \rho\right)+K_{0}\left(\xi_{2} \rho\right) I_{1}\left(\xi_{2} b\right) / K_{1}\left(\xi_{2} b\right)$ and $F_{1}\left(\xi_{2} \rho\right)=-I_{1}\left(\xi_{2} \rho\right)+$ $K_{1}\left(\xi_{2} \rho\right) I_{1}\left(\xi_{2} b\right) / K_{1}\left(\xi_{2} b\right)$, being $J_{0}, J_{1}, I_{0}, I_{1}, K_{0}, K_{1}$ the corresponding Bessel and Hankel functions.

The normalized stored energy, $W_{0}$, can be written as $W_{0}=W_{\text {int }}(1+R)$, where $R$ is the ratio of the energy stored outside the dielectric rod to that stored inside, and can be written as [10]:

$$
R=\frac{1}{\varepsilon_{r}}\left[\frac{\xi_{1} J_{0}\left(\xi_{1} a\right)}{\xi_{2} K_{0}\left(\xi_{2} a\right)}\right]^{2} \frac{\int_{a}^{b} K_{1}^{2}\left(\xi_{2} \rho\right) \rho \mathrm{d} \rho}{\int_{0}^{a} J_{1}^{2}\left(\xi_{1} \rho\right) \rho \mathrm{d} \rho}
$$

and $W_{\text {int }}$ is the normalized stored energy inside the dielectric and can be written as:

$$
W_{\mathrm{int}}=\frac{\varepsilon_{0} \varepsilon_{r} \pi^{5} h}{2}\left(\frac{240}{\xi_{1} \lambda_{0}}\right)^{2} \int_{0}^{a} J_{1}^{2}\left(\xi_{1} \rho\right) \rho \mathrm{d} \rho
$$

where $h$ is the height of the dielectric and $\lambda_{0}$ the vacuum wavelength.

By applying equations (5)-(7) to (4) we can find the third order intermodulation power coupled out of the resonator for given values of $\Delta R_{\alpha}, \Delta L_{\alpha}$ and $\alpha$. 
Table 1. Relevant parameters of the set up used for the unpatterned characterization.

\begin{tabular}{lllllllll}
\hline Region & $Q_{0}$ & $\kappa$ & $f_{0}(\mathrm{GHz})$ & $W_{0}\left(\mathrm{H} \mathrm{m}^{2}\right)$ & $\Gamma_{\alpha}\left(\mathrm{m}^{2}\right)$ & $T_{\alpha}$ & $\alpha$ & $j_{\mathrm{IMD}}\left(\mathrm{A} \mathrm{m} \mathrm{m}^{-2}\right)$ \\
\hline 1 & 12300 & 0.54 & 8.18 & $4.52 \times 10^{-15}$ & $3.01 \times 10^{-7}$ & 0.3617 & 1.2 & $0.54 \times 10^{10}$ \\
2 & 12300 & 0.54 & 8.18 & $4.52 \times 10^{-15}$ & $9.36 \times 10^{-7}$ & 0.0252 & 0.1 & $1.53 \times 10^{15}$ \\
\hline
\end{tabular}

\subsection{Half-wave resonant transmission line}

In this case we cannot apply (4) directly since the field distribution $f(\vec{r})$ in these structures (microstrip, coplanar or stripline) cannot be obtained with closed-form equations due to the sharp current peaks in the cross-sections of these transmission lines.

Nevertheless, the equivalent circuit of a transmission line using distributed RLCG parameters can be used [11], which results in a longitudinal description of the problem where $f(z)$ is the current distribution along the line for the resonant mode. That is, for a half wave open-ended resonator extending from $z=0$ to $l: f(z)=\sin (\pi z / l)$, where $l=\lambda / 2$ is the length of the line.

The nonlinear effects of HTS lines can be modelled by the dependence of their resistance and inductance per unit length on the total current through the line $(R(i), L(i))$ [5]. To be consistent with section 2 , we will use $R(i)=R_{0}+\Delta R_{\alpha}^{\prime}|i|^{\alpha}$ and $L(i)=L_{0}+\Delta L_{\alpha}^{\prime}|i|^{\alpha}$.

The voltage drop in an elemental segment of the line will be the sum of that produced by the linear elements plus the one $\left(\mathrm{d} v_{\mathrm{NL}}\right)$ produced by the nonlinear ones:

$$
\mathrm{d} v_{\mathrm{NL}}=\Delta R_{\alpha}^{\prime}|i|^{\alpha} i \mathrm{~d} z+\frac{\mathrm{d}}{\mathrm{d} t}\left(\Delta L_{\alpha}^{\prime}|i|^{\alpha} i \mathrm{~d} z\right) .
$$

We can express the current through the line as $i(z, t)=$ $\left(i_{1} \cos \omega_{1} t+i_{2} \cos \omega_{2} t\right) f(z)$ and making the analogy with previous section $\left(e_{\mathrm{NL}} \rightarrow \mathrm{d} v_{\mathrm{NL}} / \mathrm{d} z, j_{\mathrm{s}} \rightarrow i, f(\vec{r}) \rightarrow f(z)\right)$, the intermodulation power coupled out of the resonator may be obtained from equation (4), where

$$
\begin{gathered}
\Gamma_{\alpha}=0.5 \int_{0}^{l}|\sin (\pi z / l)|^{\alpha+2} \mathrm{~d} z \\
W_{0}=L_{0} l / 4 .
\end{gathered}
$$

Note that the nonlinear parameters $\left(\Delta R_{\alpha}^{\prime}\right.$ and $\left.\Delta L_{\alpha}^{\prime}\right)$, unlike the ones defined so far $\left(\Delta R_{\alpha}\right.$ and $\left.\Delta L_{\alpha}\right)$, depend on the geometry of the cross-section of the line. To evaluate these, one needs to know the volume current density distribution over the cross-section of the line [5]. The problem is somewhat complicated since this distribution depends on the penetration depth $\lambda_{L}$, and $\lambda_{L}$ is weakly dependent on the local current density. Therefore, the current distribution in the cross-section of the line depends on the total current through it, and this affects the values of $\Delta R_{\alpha}^{\prime}$ and $\Delta L_{\alpha}^{\prime}$. The steps to derive $\Delta R_{\alpha}^{\prime}$ and $\Delta L_{\alpha}^{\prime}$ from $\lambda_{L}(T, j)$ are described in [5].

\section{Measurements}

The aim of this section is to outline the steps of the procedure to compare the nonlinear parameters $\left(\alpha, j_{\mathrm{IMD}, \alpha}\right)$ extracted from an HTS sample when it is characterized before and after a patterning process.

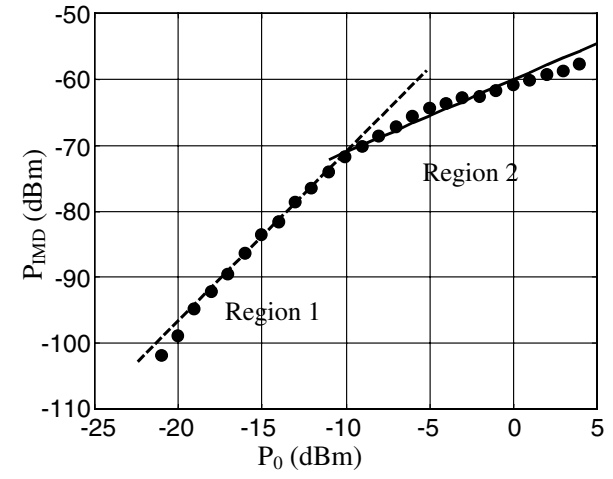

Figure 1. Black circles represent the third order intermodulation products produced by the rutile loaded cavity. The solid and dashed line are the adjustment with the closed-form equations for region 1 and 2 , respectively.

\subsection{Dielectric resonator (unpatterned sample)}

To illustrate the procedure we have made one-port intermodulation measurements in a one-sided $300 \mathrm{~nm}$ YBCO thin film (deposited on $\mathrm{MgO}$ ) using a rutile-loaded cavity. The resonator designed is like the one described in [3]: a rutile cylinder having $4 \mathrm{~mm}$ of diameter $(2 a)$ and $3 \mathrm{~mm}$ of height (h) placed on the $10 \times 10 \mathrm{~mm}^{2}$ HTS sample $(b=5)$. The resonance frequency of the cavity was $8 \mathrm{GHz}$ and the intermodulation measurements are plotted in figure 1. As done in [8], we have fed the cavity with only one port and two input tones spaced $20 \mathrm{kHz}$ apart. As shown in [3], the slope of the third order intermodulation product at $2 \omega_{1}-\omega_{2}$ sets the phenomenological parameter $\alpha$. From the measurements presented below, we see two different regions depending on the input power to the resonator. The lower power region (region 1) can be fitted with $\alpha=1.2$, whereas the higher power region (region 2) is fitted using $\alpha=0.1$.

Table 1 shows the parameters needed to obtain the nonlinear term $\left|\Delta R_{\alpha}+j \omega_{0} \Delta L_{\alpha}\right|$ to fit the intermodulation measurements of figure $1 . W_{0}$ is calculated from the dimensions of the cavity using (6) and (7). The term $\Gamma_{\alpha}$ is calculated using (5) for $\alpha=1.2$ and 0.1 . The linear parameters $Q_{0}$ and $\kappa$ are calculated from the measured scattering parameter $S_{11}$. The term $T_{\alpha, 2 \omega_{1}-\omega_{2}}$ is equal to 0.3617 for $\alpha=1.2$ and 0.0252 for $\alpha=0.1$, being $j_{1} / j_{2}=1$ since the two input tones have the same power [3]. With all of these parameters, the nonlinear term $\left|\Delta R_{\alpha}+j \omega_{0} \Delta L_{\alpha}\right|$ has to be $2.21 \times 10^{-6} \Omega \mathrm{m}^{\alpha} / \mathrm{A}^{\alpha}$ for region 1 and $0.001 \Omega \mathrm{m}^{\alpha} / \mathrm{A}^{\alpha}$ for region 2 , according to (4).

In addition, if we assume that the nonlinear contribution comes from the reactive term $\Delta L_{\alpha}$, and that the resistive term can be neglected $[2,5]$, we can calculate the phenomenological parameter $j_{\mathrm{IMD}, \alpha}$, since $\Delta L_{\alpha} \approx 0.5 \mu \lambda_{L}(T, 0) / j_{\mathrm{IMD}, \alpha, S}^{\alpha}$, as being $j_{\mathrm{IMD}, \alpha, S} \approx \lambda_{L}(T) j_{\mathrm{IMD}, \alpha}$ [14]. The resulting values are $0.54 \times 10^{10}$ and $1.53 \times 10^{15} \mathrm{~A} \mathrm{~m}^{-2}$ for $\alpha=1.2$ (region 1) and $\alpha=0.1$ (region 2), respectively. 
Comparison between nonlinear measurements in patterned and unpatterned thin films

Table 2. Relevant parameters of the set up used for the patterned characterization.

\begin{tabular}{lllllllll}
\hline$Q_{0}$ & $\kappa_{1}$ & $\kappa_{2}$ & $f_{0}(\mathrm{GHz})$ & $W_{0}(\mathrm{H})$ & $\Gamma_{\alpha}(\mathrm{m})$ & $T_{\alpha}$ & $\alpha$ & $j_{\mathrm{IMD}}\left(\mathrm{A} \mathrm{m}^{-2}\right)$ \\
\hline 700 & 2.25 & 2.25 & 6.3 & $5.23 \times 10^{-10}$ & 0.0013 & 0.0252 & 0.1 & $1.53 \times 10^{15}$ \\
\hline
\end{tabular}

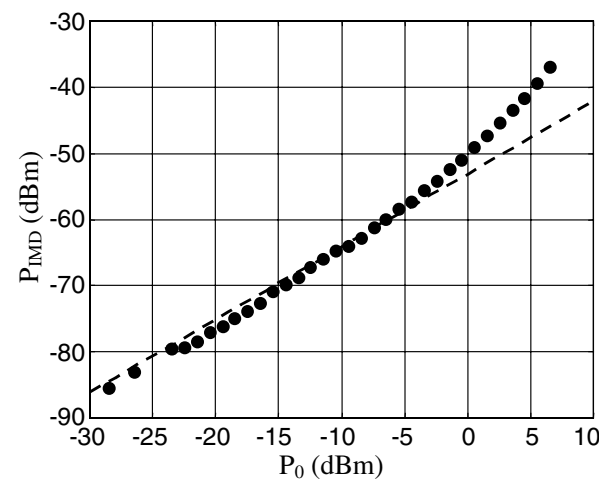

Figure 2. Black circles represent the third order intermodulation products produced by the coplanar resonant transmission line. The dashed line represents the adjustment with the closed-form equations.

\subsection{Coplanar resonator (patterned sample)}

On the same sample we have patterned a coplanar half-wave transmission line with $500 \mu \mathrm{m}$ width and $250 \mu \mathrm{m}$ gap.

In this case the resonator is a two port device and the measured linear parameters are $f_{0}=6.38 \mathrm{GHz}, Q_{0}=700$, $k_{1}=2.25$ and $k_{2}=2.25$. To perform the intermodulation measurements the resonator is fed with two tones spaced $20 \mathrm{kHz}$ apart. Figure 2 shows the intermodulation output power at $2 \omega_{1}-\omega_{2}$.

As we will see in the next section, the volume current density achieved in the transmission line at lower input power $(-30$ to $-20 \mathrm{dBm})$ is the same achieved in region 2 of the rutile loaded cavity. Therefore we use the corresponding values of $j_{\mathrm{IMD}, \alpha}$ and $\alpha$ (that is $1.53 \times 10^{15}$ and 0.1 , respectively) to obtain $\Delta R_{\alpha}^{\prime}$ and $\Delta L_{\alpha}^{\prime}$ as described in section 3.2. The resistive nonlinear parameter is $\Delta R_{\alpha}^{\prime}=16.644 \Omega /\left(\mathrm{m} \mathrm{A}^{\alpha}\right)$ and the reactive one is $\Delta L_{\alpha}^{\prime}=1.38 \times 10^{-10} \mathrm{H} /\left(\mathrm{m} \mathrm{A}^{\alpha}\right)$. From these values we see that the nonlinear resistive term is dominant over the reactive one by a factor of $4\left(\Delta R_{\alpha}^{\prime}>\omega \Delta L_{\alpha}^{\prime}\right)$. The factor $T_{\alpha, 2 \omega 1-\omega_{2}}$ remains equal to 0.0252 since again the two input tones are balanced. The terms $W_{0}$ and $\Gamma_{\alpha}$ are obtained using (9) and (10), respectively. With these values (table 2) we can use equation (4) to calculate the expected intermodulation power if no degradation exists on the sample after the etching process. The dashed line of figure 2 shows these expected values.

\subsection{Discussion}

Since the nonlinear behaviour of the superconductor depends on the volume current density, we compare the nonlinear effects in both structures as a function of the maximum volume current density reached in them. To do that, we first find the surface current density $\left(j_{\mathrm{s}}\right)$ on the unpatterned HTS film, by applying

$$
\frac{\omega_{0} W_{0}}{Q_{0}}\left|j_{\mathrm{s}}\right|^{2}=4 P_{0} \frac{\kappa}{(1+\kappa)^{2}}
$$

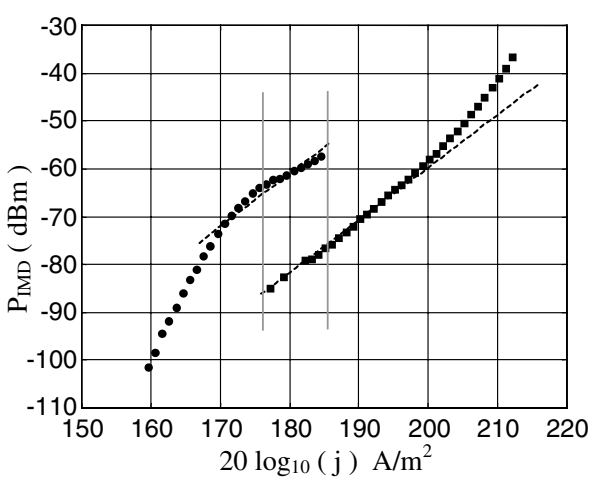

Figure 3. Black circles represent the third order intermodulation products produced by the rutile loaded cavity. Black squares represent the third order intermodulation products produced by the coplanar resonator. Both are plotted versus the peak volume current density in the superconductor. The dashed lines represent the adjustment using the nonlinear model of region 2 (see figure 1).

as done in [14]. Note that the terms $W_{0}, Q_{0}, \omega_{0}$ and $\kappa$ are the ones that appear in table 1 . The volume current density at the surface $j_{0}$ can be obtained if we consider $\vec{j}_{\mathrm{s}} \approx \lambda_{L}(T) \vec{j}_{0}[15]$.

In a two-port half-wave transmission line the maximum current, $I_{\max }$, can be found using

$$
\frac{\omega_{0} W_{0}}{Q_{0}}\left|I_{\max }\right|^{2}=4 P_{0} \frac{\kappa_{1}}{\left(1+\kappa_{1}+\kappa_{2}\right)^{2}} .
$$

Note that the terms $W_{0}, Q_{0}, \omega_{0}$ and $\kappa$ are the ones that appear in table 2. This current is distributed in the crosssection of the transmission line. To find this distribution and the maximum value at the surface, we apply the numerical method described in $[12,13]$. Figure 3 shows the previous measurements as function of the maximum current density reached in both resonators. It is clear that both measurements are consistent when the volume current density is comparable, and can be explained using the same values of $j_{\mathrm{IMD}, \alpha}$ and $\alpha$ (see tables 1 and 2).

\section{Conclusions}

We have presented procedures for determining nonlinear parameters of HTS thin films. The same type of parameter $\left(j_{\mathrm{IMD}, \alpha}\right.$ and $\alpha$ ) can be extracted from intermodulation measurements performed on either an unpatterned HTS sample mounted as an endplate in a rutile resonator cavity, or a patterned HTS coplanar waveguide. These procedures have been validated in a single HTS film, in which the values of $j_{\mathrm{IMD}, \alpha}$ and $\alpha$ that can be inferred from the cavity measurements are the same as those that result from the measurement of the coplanar waveguide. These procedures are therefore validated in a single HTS film, and should be further validated with a larger number of representative HTS samples. 


\section{Acknowledgments}

This work was supported by the Spanish Ministry of Science and Technology (MCYT) under Project MAT2002-04551 and by the Generalitat de Catalunya (DURSI) under Grant 2001 SGR 0026.

\section{References}

[1] Weinstock H and Nisenoff M 2001 Microwave Superconductivity (Netherlands: Kluwer-Academic) pp 117-48

[2] Willemsen B A, Dahm T and Scalapino D J 1997 Appl. Phys. Lett. 71 3898-900

[3] Mateu J, Collado C, Menéndez O and O'Callaghan J M 2003 Appl. Phys. Lett. 82 97-9

[4] Klein Z, Zuccaro C, Dähne U, Schulz H, Tellman N, Kutzer R, Zaitsev A G and Wördenweber R 1995 J. Appl. Phys. 78 6683

[5] Dahm T and Scalapino D J 1997 J. Appl. Phys. 81 2002-9
[6] Willemsen B A, Kihlstrom K E, Dahm T, Scalapino D J, Gowe B, Bonn D A and Hardy W N 1998 Phys. Rev. B 58 $6650-5$

[7] Dahm T and Oppenländer J 2001 IEEE Trans. Appl. Supercond. 11 1392-5

[8] Mateu J, Collado C, Menéndez O and O'Callaghan J M 2003 J. Supercond. 16 873-80

[9] Mateu J, Collado C and O'Callaghan J M 2001 IEEE Trans. Appl. Supercond. 11 135-8

[10] Mazierska J and Grabovickic R 1998 IEEE Trans. Appl. Supercond. 8 178-87

[11] Pozar D 1998 Microwave Engineering (New York: Wiley)

[12] Weeks W T, Wu L L, McAllister M F and Singh A 1979 IBM J. Res. Dev. 23652

[13] Sheen D M and Ali S M 1991 IEEE Trans. Appl. Supercond. 1 $108-17$

[14] Mateu J 2003 Nonlinear distortion in microwave devices and systems UPC-PhD Thesis

[15] Duzer T V and Turner C W 1981 Principles of Superconductive Devices and Circuits (Amsterdam: Elsevier) 